\title{
Drivers Training based on Data from Accelerometer
}

\author{
Martin Vlkovský \\ Department of Logistics, Faculty of Military Leadership, University of Defence, \\ Brno, Czech Republic
}

\begin{abstract}
In the article, the application of modern MEMS accelerometers to evaluate driver training is discussed. Data from a transport experiment with Tatra 815 on a training polygon consisting of 4 types of surfaces were used, and the driver completed 4 individual laps. The tested parameters showed statistically significant differences between selected laps and surfaces (sections) depending on the driving style and the average speed. It is clear from the evaluation of the ride that the driver is gradually improving when driving on the polygon, as assumed. However, from a certain moment the magnitude of generated shocks exceeds normatively determined values. The design part determines specific requirements on driving characteristics of a driver during a driver training.
\end{abstract}

Keywords-driver training, transport experiment, accelerometer, statistical evaluation

\section{INTRODUCTION}

$\mathrm{T}$ RAINING of the key personnel is essential in the public and private sector. In addition, in public sector, well-prepared and well-trained workers can often save lives (e.g. health care professionals), or prevent a fatal accident (e.g. drivers).

The preparation and training of the drivers in public sector also has its specifics, especially in the military or Integrated Rescue System. In these sectors, other special requirements are imposed on drivers, such as stress resistance or ability to drive in extreme situations. Failure of a driver in such situations may result in death, injury or loss of state-owned property (or other assets) and environmental damage.

Most situations can be predicted to a certain extent and drivers can be prepared for them by appropriate preparation and training. Routine habits and automated emergency response (e.g. attacking an improvised explosive device, transport of the injured) are crucial.

New technologies are currently available as part of a training. These are mainly sophisticated simulators that enable to simulate any algorithmizable event and thus prepare drivers safely "in silico" for a dangerous or high-risk situation. For planning and securing the actual transport, simulation tools using e.g. queuing theory [1] can be applied. These approaches

The paper was written with the support of the project of long-term strategy of organization development: ROZVOLOG: Development of Capabilities and Sustainability of Logistics Support (DZRO ROZVOLOG 2016-2020) funded by the Ministry of Defence of the Czech Republic. make it possible to prepare drivers safely and relatively cheaply for both standard and specific situations and to avoid bottlenecks in the transport organization and prepare suitable scenarios for the drivers in advance.

Modern technologies for data collection of psychophysiological parameters of drivers (ECG, EEG etc. [2]), or technical parameters of the vehicle (e.g. acceleration) are used as well. The article focuses on the use of modern MEMS accelerometers with datalogger with high accuracy, which will enable, using suitable statistical tools, evaluation of the ride of a truck driver in terms of the shocks affecting the vehicle, driver and cargo. Data evaluation enables not only the evaluation of individual rides (e.g. [3]) of the respective driver, therefore determining whether the ride complies with general assumptions or specific requirements of an employer and whether the driver improves/stagnates/deteriorates, but above all, it is a tool to support a choice of suitable training methods and directing the driving style of drivers.

The application of "hard" data and quantitative methods enables an objective evaluation of the selected parameters of a ride. The examined acceleration values (shocks), which are recorded by the measuring device (accelerometer) in the form of acceleration coefficients (multiples of normal gravity acceleration), allow to detect primarily the existence of extreme values exceeding the normatively determined values of acceleration coefficient (see below) and also detect deviations in the driving style, which could have a negative impact not only on the transported cargo, but also on the driver himself or on the vehicle and its individual parts.

Considering cargo, the knowledge of anticipated magnitude of the inertia forces affecting the cargo during transportation is crucial. The inertia forces are based on the shocks (values of the acceleration coefficients), to which the magnitude of locking forces of the respective fastening system must correspond [4]. In other words, the fastening method depends (among others) on the given assumption. The magnitude of the inertia forces can be determined experimentally, which is laborious, time-consuming and therefore impractical for normal use. For this reason, values of the acceleration coefficients from the corresponding standards are used. In Europe, including the Czech Republic, it is the standard Czech State Standard (CSN) - European Standard (EN) 121951:2011. The standard CSN EN 12195-1:2011 contains 
principles and calculations for cargo securing (Cargo Securing) and presents empirically determined acceleration coefficients (for the $x, y$ and $z$ axes: 0.8, 0.6 and 1.0) and formulas for the calculation of various fastening methods [5]. The standard CSN EN 12195-1:2011 is followed by examples of EU good practice prepared by Directorate General for Energy and Transport of the European Commission, 2014, European Best Practice Guidelines on Cargo Securing for Road Transport [6]. The issue of cargo securing is further dealt with in a monograph by T. Lerher, 2015, Cargo Securing in Road Transport Using Restraining Method with Top-Over Lashing. The author of the monograph deals with general principles of cargo securing in road transport and also with the fastening of the cargo using lashing straps, so called Top-Over Lashing [7]. Another relevant monograph is a book by G. Grossmann and M. Kassmann, 2007, Safe Packaging and Load Securing in Transport, which in addition to packing functions presents cargo securing methods, including models for topover lashing using lashing straps [8].

\section{DATA AND METHODS}

\section{A. Data}

Data for the statistical analysis were obtained during a transport experiment in the military training area VyskovBrezina (Czech Republic) on a polygon consisting of 4 different types of surfaces (see Figure 1):

- Blue - Muddy Terrain (length: 1,150 m) - 1. section,

- Green - Gravel (length: 500 m) - 2. section,

- Yellow - Granite Cubes (length: 450 m) - 3. section,

- Red - Unpaved Road (length: 450 m) - 4. section.

The total route length - test polygon was 2,550 meters. A professional driver - soldier drove on the test polygon $4 \times$ without restrictions, respecting all the general security principles, i.e. there was a presumption of gradual improvement (acceleration) within the sections due to better acquitance with the conditions of the given route with each additional lap. In connection with this, the average speed was measured within a given surface (transport route type) and within the whole circuit. The magnitude of shocks (values of the acceleration coefficients) is proportionally affected by speed. Recording of the instant speed does not offer, due to shocks, sufficient accuracy and therefore the average speed was used.

The test vehicle was an off-road truck Tatra 815 VVN $6 \times 6$ (hereinafter referred to as "Tatra 815”) without cargo, which is designed for the transport of persons and cargo at maximum weight of 8,000 kg [9].

Data (see Figure 2) were measured on a measuring device accelerometer with a datalogger and OMEGA-CPULTRASHOCK-5 calibration certificate with a measuring range of $\pm 5 g$.

The measuring device recorded the highest or the lowest value each second with a sampling frequency of $512 \mathrm{~Hz}$. The $z$-axis is shifted by $1 g$ (normal gravity acceleration value).

The transport experiment was conducted under difficult conditions at the end of February during the field training exercise of students of the University of Defence in Brno. The outdoor temperature was several degrees above 0 and especially the off-road section (blue route section) was waterlogged with snow residues, which the driver had to take into account when driving. Visibility was very good and no rain or snowfall was recorded during the transport experiment.

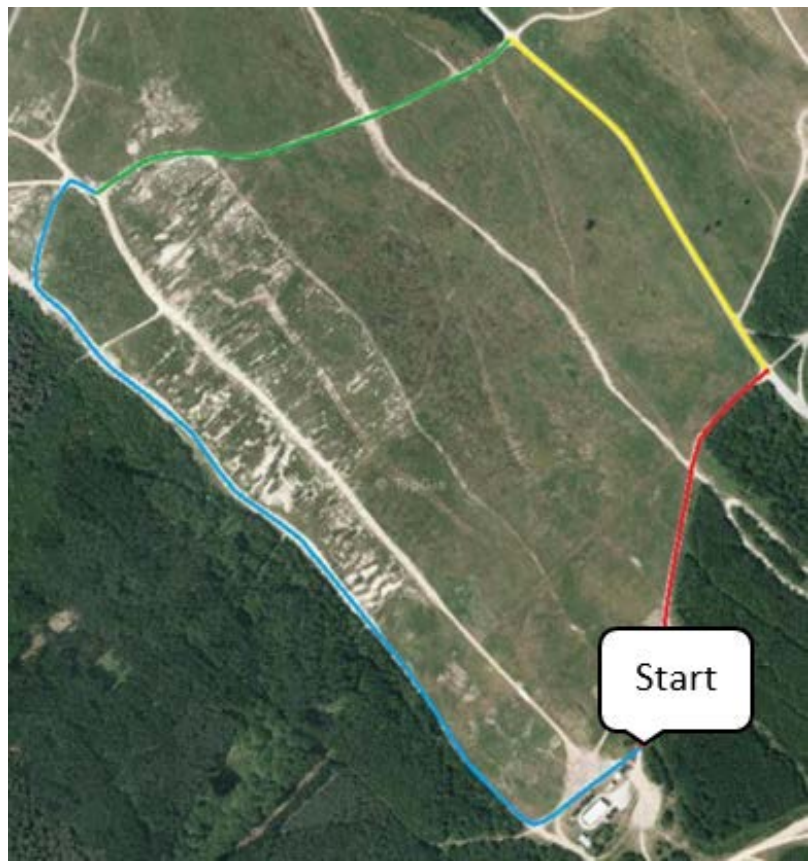

Fig. 1. Polygon

Source: Google Maps

In total 6,063 data units were obtained - acceleration coefficient values (2,021 for each axis). The data were further divided into 4 separate datasets for each lap (total of 16 subdatasets). Datasets are marked formally chronologically $d_{1}-d_{4}$. In each dataset, individual sections - types of surfaces according to colors in Figure 1, are further distinguished:

- Blue - Muddy Terrain, index B,

- Green - Gravel, index G,

- Yellow - Granite Cubes, index Y,

- Red - Unpaved Road, index R.

The numbers of data in individual sections with the corresponding marking are in Table 1.

Table1. Data of Particular Datasets

\begin{tabular}{|l|r|c|c|c|}
\cline { 2 - 5 } \multicolumn{1}{c|}{} & \multicolumn{4}{c|}{ DATASET } \\
\hline ROAD & \multicolumn{1}{c|}{$d_{1}$} & $d_{2}$ & $d_{3}$ & \multicolumn{1}{c|}{$d_{4}$} \\
\hline Blue - Muddy Terrain & 375 & 338 & 311 & 305 \\
\hline Green - Gravel & 96 & 69 & 68 & 64 \\
\hline Yellow - Granite Cubes & 49 & 47 & 50 & 46 \\
\hline Red - Unpaved Road & 55 & 52 & 49 & 47 \\
\hline TOTAL & 575 & 506 & 478 & 462 \\
\hline
\end{tabular}




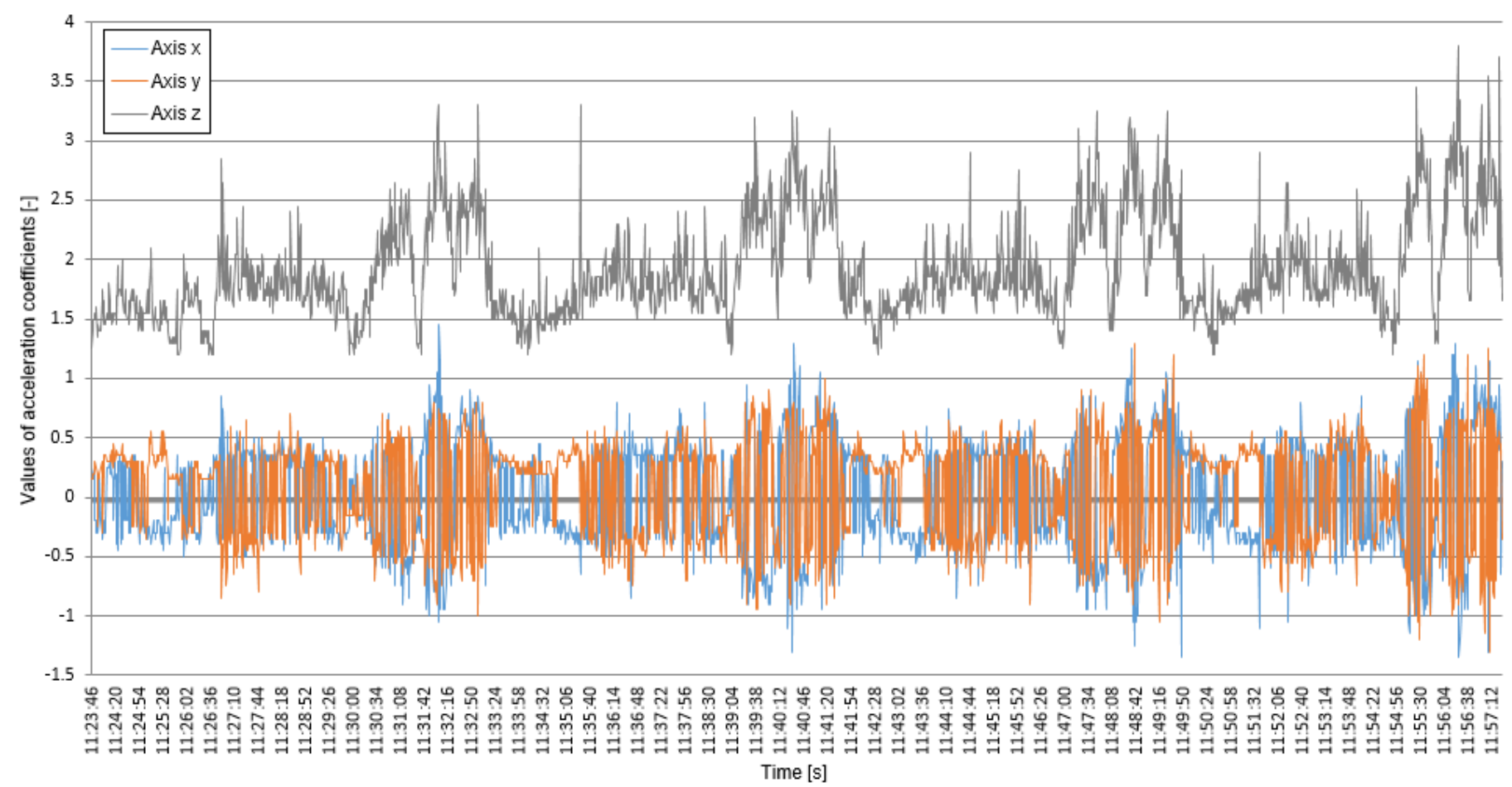

Fig. 2. Raw Data from Accelerometer

\section{B. Methods}

In order to obtain primary data, the corresponding transport experiment was carried out under the required conditions, which allowed creating relevant datasets (per lap and per each section). The measuring device was recording the acceleration coefficients (multiples of normal gravity acceleration) in three axes - longitudinal $(x)$, transverse $(y)$ and vertical ( $z$ ). Every second an entry was made per each axis, therefore the number of data also determined the duration of the transport experiment, i.e. time needed for each lap or section. From here, it was possible to calculate the average speed within each lap or section.

Basic descriptive characteristics were used to describe and evaluate the individual laps: the arithmetic mean of the absolute values of the measured coefficients of acceleration, variance, skewness and kurtosis. The absolute values of acceleration coefficients for the arithmetic mean were used for the analysis of the magnitude of the shocks, not their direction (oscillation around the corresponding values 0 ).

Tests of statistical hypotheses intended for the corresponding laps or sections were performed at the significance level $\alpha=0.05$ using statistical tests of equality of the mean values, i.e. variance.

The normality was verified graphically by Q-Q plots and although slight deviations from the normality were detected, the theoretical and empirical quantiles were approximately on one straight line.
For the testing purpose, null $(H)$ and alternative hypothesis (A) were formulated, separately for whole laps $\left(H_{1}\right.$ and $\left.A_{1}\right)$ and for particular surfaces $\left(\mathrm{H}_{2}\right.$ and $\left.A_{2}\right)$ :

- $H_{1}$ : The mean values and variations of acceleration coefficients measured at T-815 on first lap are not statistically significantly different from last lap.

The appropriate pairs of laps are tested: $d_{1}-d_{2}, d_{1}-d_{3}, d_{1}-d_{4}$, $d_{2}-d_{3}, d_{2}-d_{4}$ and $d_{3}-d_{4}$. The first $\left(d_{1}\right)$ and last lap $\left(d_{4}\right)$ are considered as two extreme cases and are suject of further analysis (hypotheses $\mathrm{H}_{2}$ and $\mathrm{A}_{2}$ ).

- $A_{1}$ : The mean values and variations of acceleration coefficients measured at T-815 on particular lap are lower than on following lap.

It is assumed that driver will improve his ride after each lap due a better knowledge of particular route and will accelerate.

- $H_{2}$ : The mean values and variations of acceleration coefficients measured at T-815 at first lap $\left(d_{1}\right)$ on particular surface are not statistically significantly different from another one measured at last lap $\left(d_{4}\right)$.

The following pairs of surfaces on two extreme laps ( $d_{1}$ and $d_{4}$ ) are tested: $d_{1 B}-d_{1 G}, d_{1 B}-d_{1 Y}, d_{1 B}-d_{1 R}, d_{1 G}-d_{1 Y}, d_{1 G}-d_{1 R}$, $d_{1 Y}-d_{1 R}, d_{4 B}-d_{4 G}, d_{4 B}-d_{4 Y}, d_{4 B}-d_{4 R}, d_{4 G}-d_{4 Y}, d_{4 G}-d_{4 R}, d_{4 Y}-d_{4 R}$, $d_{1 B}-d_{4 B}, d_{1 B}-d_{4 G}, d_{1 B}-d_{4 Y}, d_{1 B}-d_{4 R}, d_{1 G}-d_{4 B}, d_{1 G}-d_{4 G}, d_{1 G}-d_{4 Y}$, $d_{1 G}-d_{4 R}, d_{1 Y}-d_{4 B}, d_{1 Y}-d_{4 G}, d_{1 Y}-d_{4 Y}, d_{1 Y}-d_{4 R}, d_{1 R}-d_{4 B}, d_{1 R}-d_{4 G}$, $d_{1 R}-d_{4 Y}$ and $d_{1 R}-d_{4 R}$.

- $A_{2}$ : The mean values and variations of acceleration coefficients measured at T-815 at first lap $\left(d_{1}\right)$ on particular surface are statistically significantly different from another one measured at last lap $\left(d_{4}\right)$.

It is assumed that worse surfaces will generate higher shocks (values of acceleration coefficients). 
To verify the hypothesis $H_{1}$, partial hypothesis (six tests) were separately tested for individual axes and each of the tested characteristics. A right-hand test was used to test the alternative hypothesis $A_{1}$. Tab. 2 shows the appropriate statistical evaluation of alternative hypothesis $A_{1}$.

The testing was performed using the critical region, calculating the value of the test statistic and comparing it with the relevant critical region. If the value of the test statistic falls into the critical region, the null hypothesis $H_{1}$ is rejected at the significance level $\alpha$. For all tests, significance level $\alpha=0.05$ was used.

To verify the hypothesis $H_{2}$, partial hypothesis (28 tests) were separately tested for individual axes and both of the tested characteristics. A right-hand test was used to test the alternative hypothesis $A_{2}$. Tab. 2 shows the appropriate statistical evaluation of alternative hypothesis $A_{2}$.

The testing was performed analogously to $H_{1}$ and the used significance level was the same too $(\alpha=0.05)$.

On the basis of measurement and expert estimation, my own propositions were made in the conclusion in the form of best practices to ensure safe cargo securing.

Statistical evaluation of the measured data was firstly carried out for the whole datasets $\left(d_{1}-d_{4}\right)$ and secondly for the sub-sections (successively on individual surfaces within the given dataset). From the overall datasets a conclusion can be drawn in relation to the improvement of the ride in general, i.e. the improvement due to better acquitance with the route and training. Within the sub-sections it is possible to evaluate movement and improvement of the driver on individual surfaces - types of transport routes, or adapt the driving to particularities of the given transport route in the context of climatic conditions.

\section{RESULTS AND DISCUSSIONS}

\section{A. Evaluation of whole datasets}

The differences in driving during the individual laps can be demonstrated either with the data in Table 1 or in Figure 3, where the individual datasets are shown in a color scale (laps). The first round is marked with the darkest color and the successive rounds are progressively marked with lighter colors.

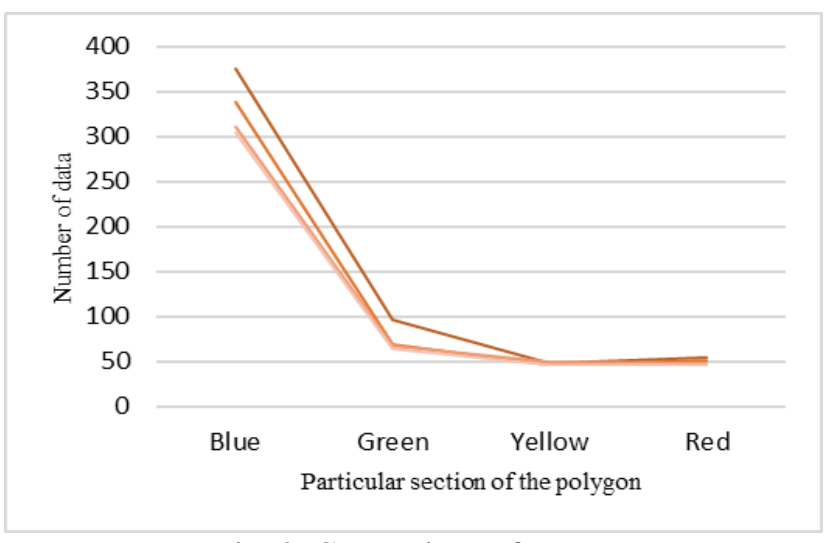

Fig. 3. Comparison of Datasets
Table 1 shows that the driver drives faster in each round due to the acquitance with the route and the specific terrain conditions. The number of data due to an entry every second also determines the time in which a driver went around the polygon. In percentage terms the driver needed, in comparison with the first round $\left(d_{1}, 100 \%\right), 88 \%$ of that time in the second round $\left(d_{2}\right), 83 \%$ in the third $\left(d_{3}\right), 80 \%$ in the fourth $\left(d_{4}\right)$. This corresponds to the average speed in individual laps respectively: $4.43 \mathrm{~m} \cdot \mathrm{s}^{-1}$ in the first round, $5.04 \mathrm{~m} \cdot \mathrm{s}^{-1}$ in the second, $5.33 \mathrm{~m} \cdot \mathrm{s}^{-1}$ in the third, $5.52 \mathrm{~m} \cdot \mathrm{s}^{-1}$ in the fourth.

The first dataset (first round, $d_{1}$ ) does not show extreme values for the basic descriptive characteristics. Regarding the extreme values of the acceleration coefficients in the individual axes, a total of 209 values exceeding the normatively prescribed limits according to CSN EN 12195$1: 2011$ (141 of them in the $z$-axis) were found, representing $12.12 \%$ of the total measured values (575 values). Only 3 values (also in the $z$-axis) exceeded the prescribed limit by more than double, which represents $0.17 \%$ of the total number of measured values.

The second dataset (second round, $d_{2}$ ) does not show extreme values for the basic descriptive characteristics. However, the mean value (arithmetic mean of absolute values) of the $z$-axis is closer to the normatively prescribed limit than the previous dataset (value 1.9090 compared to value 1.8196 for $d_{1}$ ). Regarding the extreme values of acceleration coefficients in the individual axes, a total of 247 values exceeding the prescribed limits according to CSN EN 121951:2011 were measured (152 of them in the $z$-axis), representing $16.27 \%$ of the total measured values (506 values). Only 5 values (also in the $z$-axis) exceeded normatively prescribed limit more than double, representing $0.33 \%$ of the total number of measured values.

The third dataset (third round, $d_{3}$ ) does not show extreme values for the basic descriptive characteristics. The mean value (arithmetic mean of absolute values) for the $z$-axis is close to the normatively determined limit (value 1.9487 compared to the value 1.9090 for $d_{2}$ ). Regarding the extreme values in the individual axes, a total of 240 values exceeding normatively prescribed limits according to CSN EN 12195-1:2011 (152 of them in the $z$-axis) were found, representing $16.74 \%$ of the total number of measured values (478 values). Only 5 values (4 of them in the $z$-axis) exceeded the prescribed limit more than double, which represents $0.35 \%$ of the total number of measured values.

The fourth dataset (fourth round, $d_{4}$ ) does not show extreme values for the basic descriptive characteristics. The mean value (arithmetic mean of absolute values) for the $z$-axis is close to the normatively determined limit (value 1.9834 compared to the value 1.9487 for $d_{3}$ ). Regarding the extreme values of acceleration coefficients in the individual axes, a total of 304 values exceeding the normatively prescribed limits according to CSN EN 12195-1:2011 (161 of them in the $z$-axis) were measured, which represents $21.93 \%$ of the total measured values (462 values). Only 14 values (13 of them in the $z$-axis) 
exceeded the normatively prescribed limit more than double, representing $1.01 \%$ of the total number of the measured values.

Overall, based on the evaluation of all 4 datasets, the basic descriptive characteristics do not show significant anomalies with the exception of the mean value (arithmetic mean of absolute values), which is very close to the normatively determined limit of $2.0 \mathrm{~g}$, especially for $d_{4}$ and point to the hazardous cargo securing in the $z$-axis. Each of the datasets contains relatively non-negligible percentage of values, which exceeds the normatively determined values of the acceleration coefficients according to CSN EN 12195-1:2011, which may be potentially hazardous. The $z$-axis is prevalent in all these cases. A higher occurrence of values exceeding the normatively determined limits more than double can be considered potentially hazardous. In the fastest, fourth round $\left(d_{4}\right)$, where the occurrence of these values is the highest, more than $1 \%$ of the values exceed by double.

Furthermore, the individual datasets, i.e. the data in the corresponding axes, were compared. Two-tailed tests and subsequently one-tailed tests were performed to determine whether there was a statistically significant difference at the significance level $\alpha=0.05$ between the compared datasets, or whether values in some datasets are larger/smaller than in others. The results are summarized in Table 2.

Table 2. Statistical tests $-d_{1}-d_{4}$

\begin{tabular}{|l|c|c|c|c|c|c|}
\cline { 2 - 7 } \multicolumn{1}{c|}{} & \multicolumn{3}{c|}{$\mu_{i}$ (ABS) } & \multicolumn{3}{c|}{$\sigma_{i}{ }^{2}$} \\
\cline { 2 - 7 } \multicolumn{1}{c|}{} & $c_{x}$ & $c_{y}$ & $c_{z}$ & $c_{x}$ & $c_{y}$ & $c_{z}$ \\
\hline $\begin{array}{l}\boldsymbol{d}_{1}- \\
\boldsymbol{d}_{2}\end{array}$ & $\mu_{1}<\mu_{2}$ & $\mu_{1}<\mu_{2}$ & $\mu_{1}<\mu_{2}$ & $\sigma_{1}{ }^{2}<\sigma_{2}{ }^{2}$ & $\sigma_{1}{ }^{2}<\sigma_{2}{ }^{2}$ & $N O$ \\
\hline $\begin{array}{l}\boldsymbol{d}_{1}- \\
\boldsymbol{d}_{3}\end{array}$ & $N O$ & $\mu_{1}<\mu_{3}$ & $N O$ & $\sigma_{1}{ }^{2}<\sigma_{3}{ }^{2}$ & $\sigma_{1}{ }^{2}<\sigma_{3}{ }^{2}$ & $N O$ \\
\hline $\begin{array}{l}\boldsymbol{d}_{1^{-}} \\
\boldsymbol{d}_{4}\end{array}$ & $\mu_{1}<\mu_{4}$ & $\mu_{1}<\mu_{4}$ & $\mu_{1}<\mu_{4}$ & $\sigma_{1}{ }^{2}<\sigma_{4}{ }^{2}$ & $\sigma_{1}{ }^{2}<\sigma_{4}{ }^{2}$ & $\sigma_{1}{ }^{2}<\sigma_{4}{ }^{2}$ \\
\hline $\begin{array}{l}\boldsymbol{d}_{2}- \\
\boldsymbol{d}_{3}\end{array}$ & $N O$ & $N O$ & $N O$ & $N O$ & $N O$ & $N O$ \\
\hline $\begin{array}{l}\boldsymbol{d}_{2}- \\
\boldsymbol{d}_{4}\end{array}$ & $\mu_{2}<\mu_{4}$ & $\mu_{2}<\mu_{4}$ & $\mu_{2}<\mu_{4}$ & $\sigma_{2}{ }^{2}<\sigma_{4}{ }^{2}$ & $\sigma_{2}{ }^{2}<\sigma_{4}{ }^{2}$ & $\sigma_{2}{ }^{2}<\sigma_{4}{ }^{2}$ \\
\hline $\begin{array}{l}\boldsymbol{d}_{3}- \\
\boldsymbol{d}_{4}\end{array}$ & $\mu_{3}<\mu_{4}$ & $\mu_{3}<\mu_{4}$ & $N O$ & $\sigma_{3}{ }^{2}<\sigma_{4}{ }^{2}$ & $\sigma_{3}{ }^{2}<\sigma_{4}{ }^{2}$ & $\sigma_{3}{ }^{2}<\sigma_{4}{ }^{2}$ \\
\hline
\end{tabular}

It is clear from Table 2, that the statistically significant differences at the significance level $\alpha=0.05$ for the two observed parameters (arithmetic mean of the absolute values of the acceleration coefficeitns and variance) were observed in the datasets $d_{1}-d_{4}$ and $d_{2}-d_{4}$. The results also show a great similarity in the pair of datasets $d_{2}-d_{3}$ and partially also $d_{1}-d_{3}$. Overall, great differences between the first and the last round can be drawn from the results, when it is clear that during the last round the driver drove significantly faster due to the better acquitance with the terrain, and the test vehicle generated greater shocks. Given the difference in the percentage representation of the values of the acceleration coefficients exceeding the normatively determined values in the pair of datasets $d_{1}-d_{4}$, almost 10 percentage points, the insignificant representation of values exceeding the normatively determined values by double for $d_{4}$, the last round can be considered risky or potentially hazardous in terms of cargo securing. In terms of cargo securing the last round can be considered unsatisfactory regarding the driving technique and the average speed of the vehicle.

From the above it can be stated that $H_{1}$ is rejected and $A_{1}$ is accepted for the extremes (first lap $-d_{1}$ and last lap $-d_{4}$ ). The $H_{1}$ is rejected even for other pair $\left(d_{2}-d_{4}\right)$. In average, shocks (influencing vehicle, driver and cargo) were higher in the last lap than in the first one (second one).

Based on the results of the overall evaluation (all 4 datasets), the individual sections - transport routes will be compared for the two extreme cases $d_{1}$ and $d_{4}$.

\section{B. Evaluation of individual sections}

For the purpose of evaluation of individual sections the two extreme cases were chosen - the first and the last round (lap). For completeness, all pairs of datasets were compared, i.e. datasets of the individual sections. Statistical tests were performed at the significance level $\alpha=0.05$ and it was a total of 22 compared pairs of datasets. The results of the statistical tests for the individual sections (types of transport routes) are summarized in Table 3, where the indexes mark the corresponding section (see above).

The individual sections were first compared within the given dataset $\left(d_{1}\right.$, and $\left.d_{4}\right)$ - see the first 12 rows of Table 3 . The results imply that both datasets show statistically significant difference at the significance level $\alpha=0.05$ between the segments BLUE - GREEN, BLUE - YELLOW and BLUE RED for both observed parameters.

The situation for another type of transport route - section GREEN was similar, although the statistical significance was confirmed only for $d_{1}$ for the arithmetic mean of absolute values of the acceleration coefficients and for $d_{4}$ in the axes $x$ and $z$, the segment GREEN is clearly different from the compared segments (YELLOW and RED). The last pair (YELLOW and RED) either did not show statistically significant difference for both datasets or 3 results ( $x$-axis for $d_{1}$ for both parameters, or $x$-axis for $d_{4}$ for variance) were to the detriment of the YELLOW section. Therefore, it is possible to conclude that the properties in the terms of shocks are worse in the YELLOW segment than in the RED segment.

In the next part (Table 3, rows 13-28) a pair comparison of all sections for both observed datasets was performed ( $d_{1}$ and $\left.d_{4}\right)$.

The comparison monitors both the difference between sections and between the two datasets, assuming that the last section generated greater shocks due to higher average speed in all sections.

The section speed was for the monitored datasets as follows:

- BLUE $-d_{1}-3.07 \mathrm{~m} \cdot \mathrm{s}^{-1}, d_{4}-3.77 \mathrm{~m} \cdot \mathrm{s}^{-1}$,

- GREEN $-d_{1}-5.21 \mathrm{~m} \cdot \mathrm{s}^{-1}, d_{4}-7.81 \mathrm{~m} \cdot \mathrm{s}^{-1}$,

- YELLOW $-d_{1}-9.18 \mathrm{~m} \cdot \mathrm{s}^{-1}, d_{4}-9.78 \mathrm{~m} \cdot \mathrm{s}^{-1}$,

- RED $-d_{1}-8.18 \mathrm{~m} \cdot \mathrm{s}^{-1}, d_{4}-9.57 \mathrm{~m} \cdot \mathrm{s}^{-1}$. 
Table 3. Statistical evaluation - individual sections (types of transport routes)

\begin{tabular}{|c|c|c|c|c|c|c|c|}
\hline \multirow[b]{2}{*}{ No. } & \multirow[b]{2}{*}{ Datasets pair } & \multicolumn{3}{|c|}{$\mu_{i}(A B S)$} & \multicolumn{3}{|c|}{$\sigma_{i}^{2}$} \\
\hline & & $c_{x}$ & $c_{y}$ & $c_{z}$ & $c_{x}$ & $c_{y}$ & $c_{z}$ \\
\hline 1 & $d_{1 B}-d_{1 G}$ & $\mu_{1 B}<\mu_{1 G}$ & $\mu_{1 B}<\mu_{1 G}$ & $\mu_{1 B}<\mu_{1 G}$ & $\sigma_{1 B}{ }^{2}<\sigma_{1 G}{ }^{2}$ & $\sigma_{1 B}{ }^{2}<\sigma_{1 G}{ }^{2}$ & $\sigma_{1 B}{ }^{2}<\sigma_{1 G}{ }^{2}$ \\
\hline 2 & $d_{1 B}-d_{1 Y}$ & $\mu_{1 B}<\mu_{1 Y}$ & $\mu_{1 B}<\mu_{1 Y}$ & $\mu_{1 B}<\mu_{1 Y}$ & ${\sigma_{1 B}{ }^{2}<{\sigma_{1 Y}}^{2}}^{2}$ & ${\sigma_{1 B}^{2}<\sigma_{1 Y}^{2}}^{2}$ & ${\sigma_{1 B}}^{2}<{\sigma_{1 Y}}^{2}$ \\
\hline 3 & $d_{1 B}-d_{1 R}$ & $\mu_{1 B}<\mu_{1 R}$ & $\mu_{1 B}<\mu_{1 R}$ & $\mu_{1 B}<\mu_{1 R}$ & ${\sigma_{1 B}{ }^{2}<\sigma_{1 R}{ }^{2}}^{2}$ & ${\sigma_{1 B}{ }^{2}<\sigma_{1 R}^{2}}^{2}$ & ${\sigma_{1 B}{ }^{2}<\sigma_{1 R}{ }^{2}}^{2}$ \\
\hline 4 & $d_{1 G}-d_{1 Y}$ & $\mu_{1 G}<\mu_{1 Y}$ & $\mu_{1 G}<\mu_{1 Y}$ & $\mu_{1 G}<\mu_{1 Y}$ & $\sigma_{1 G}{ }^{2}<\sigma_{1 Y}{ }^{2}$ & $\sigma_{1 G}{ }^{2}<\sigma_{1 Y}{ }^{2}$ & NO \\
\hline 5 & $d_{1 G}-d_{1 R}$ & $\mu_{1 G}<\mu_{1 R}$ & $\mu_{1 G}<\mu_{1 R}$ & $\mu_{1 G}<\mu_{1 R}$ & NO & $\sigma_{1 G}^{2}<\sigma_{1 R}^{2}$ & NO \\
\hline 6 & $d_{1 Y}-d_{1 R}$ & $\mu_{1 Y}>\mu_{1 R}$ & NO & NO & ${\sigma_{1 Y}{ }^{2}>\sigma_{1 R}{ }^{2}}^{2}$ & $N O$ & NO \\
\hline 7 & $d_{4 B}-d_{4 G}$ & $\mu_{4 B}<\mu_{4 G}$ & $\mu_{4 B}<\mu_{4 G}$ & $\mu_{4 B}<\mu_{4 G}$ & ${\sigma_{4 B}{ }^{2}<\sigma_{4 G}{ }^{2}}^{2}$ & ${\sigma_{4 B}}^{2}<\sigma_{4 G}{ }^{2}$ & ${\sigma_{4 B}}^{2}<{\sigma_{4 G}}^{2}$ \\
\hline 8 & $d_{4 B}-d_{4 Y}$ & $\mu_{4 B}<\mu_{4 Y}$ & $\mu_{4 B}<\mu_{4 Y}$ & $\mu_{4 B}<\mu_{4 Y}$ & $\sigma_{4 B}{ }^{2}<\sigma_{4 Y}{ }^{2}$ & $\sigma_{4 B}{ }^{2}<\sigma_{4 Y}{ }^{2}$ & $\sigma_{4 B}{ }^{2}<\sigma_{4 Y}{ }^{2}$ \\
\hline 9 & $d_{4 B}-d_{4 R}$ & $\mu_{4 B}<\mu_{4 R}$ & $\mu_{4 B}<\mu_{4 R}$ & $\mu_{4 B}<\mu_{4 R}$ & $\sigma_{4 B}{ }^{2}<\sigma_{4 R}{ }^{2}$ & $\sigma_{4 B}{ }^{2}<\sigma_{4 R}{ }^{2}$ & $\sigma_{4 \mathrm{~B}}{ }^{2}<\sigma_{4 R}{ }^{2}$ \\
\hline 10 & $d_{4 G}-d_{4 Y}$ & $\mu_{4 G}<\mu_{4 Y}$ & NO & $\mu_{4 G}<\mu_{4 Y}$ & NO & NO & NO \\
\hline 11 & $d_{4 G}-d_{4 R}$ & $\mu_{4 G}<\mu_{4 R}$ & NO & $\mu_{4 G}<\mu_{4 R}$ & NO & NO & NO \\
\hline 12 & $d_{4 Y}-d_{4 R}$ & NO & $\mathrm{NO}$ & NO & $\sigma_{4 Y}^{2}>\sigma_{4 R}^{2}$ & NO & NO \\
\hline 13 & $d_{1 B}-d_{4 B}$ & $\mu_{1 B}<\mu_{4 B}$ & $\mu_{1 B}<\mu_{4 B}$ & $\mu_{1 B}<\mu_{4 B}$ & $\sigma_{1 B}^{2}<\sigma_{4 B}^{2}$ & $\sigma_{1 B}^{2}<\sigma_{4 B}^{2}$ & $\sigma_{1 B}{ }^{2}<\sigma_{4 B}^{2}$ \\
\hline 14 & $d_{1 B}-d_{4 G}$ & $\mu_{1 B}<\mu_{4 G}$ & $\mu_{1 B}<\mu_{4 G}$ & $\mu_{1 B}<\mu_{4 G}$ & ${\sigma_{1 B}{ }^{2}<{\sigma_{4 G}}^{2}}^{2}$ & ${\sigma_{1 B}{ }^{2}<{\sigma_{4 G}}^{2}}^{2}$ & ${\sigma_{1 B}{ }^{2}<\sigma_{4 G}{ }^{2}}^{2}$ \\
\hline 15 & $d_{1 B}-d_{4 Y}$ & $\mu_{1 B}<\mu_{4 Y}$ & $\mu_{1 B}<\mu_{4 Y}$ & $\mu_{1 B}<\mu_{4 Y}$ & $\sigma_{1 B}{ }^{2}<\sigma_{4 Y}{ }^{2}$ & $\sigma_{1 B}{ }^{2}<\sigma_{4 Y}{ }^{2}$ & $\sigma_{1 B}{ }^{2}<\sigma_{4 Y}{ }^{2}$ \\
\hline 16 & $d_{1 B}-d_{4 R}$ & $\mu_{1 B}<\mu_{4 R}$ & $\mu_{1 B}<\mu_{4 R}$ & $\mu_{1 B}<\mu_{4 R}$ & $\sigma_{1 B}{ }^{2}<\sigma_{4 R}{ }^{2}$ & $\sigma_{1 B}{ }^{2}<\sigma_{4 R}{ }^{2}$ & $\sigma_{1 B}{ }^{2}<\sigma_{4 R}{ }^{2}$ \\
\hline 17 & $d_{1 G}-d_{4 B}$ & $\mu_{1 G}>\mu_{4 B}$ & NO & $\mu_{1 G}>\mu_{4 B}$ & $N O$ & $N O$ & $\sigma_{1 G}{ }^{2}>\sigma_{4 B}{ }^{2}$ \\
\hline 18 & $d_{1 G}-d_{4 G}$ & $\mu_{1 G}<\mu_{4 G}$ & $\mu_{1 G}<\mu_{4 G}$ & $\mu_{1 G}<\mu_{4 G}$ & ${\sigma_{1 G}{ }^{2}<\sigma_{4 G}{ }^{2}}$ & $\sigma_{1 G}{ }^{2}<\sigma_{4 G}{ }^{2}$ & ${\sigma_{1 G}}^{2}<\sigma_{4 G}{ }^{2}$ \\
\hline 19 & $d_{1 G}-d_{4 Y}$ & $\mu_{1 G}<\mu_{4 Y}$ & $\mu_{1 G}<\mu_{4 Y}$ & $\mu_{1 G}<\mu_{4 Y}$ & $\sigma_{1 G}{ }^{2}<\sigma_{4 Y}{ }^{2}$ & $\sigma_{1 G}^{2}<{\sigma_{4 Y}}^{2}$ & $\sigma_{1 G}{ }^{2}<\sigma_{4 Y}{ }^{2}$ \\
\hline 20 & $d_{1 G}-d_{4 R}$ & $\mu_{1 G}<\mu_{4 R}$ & $\mu_{1 G}<\mu_{4 R}$ & $\mu_{1 G}<\mu_{4 R}$ & $\sigma_{1 G}^{2}<\sigma_{4 R}^{2}$ & $\sigma_{1 G}^{2}<\sigma_{4 R}^{2}$ & NO \\
\hline 21 & $d_{1 Y}-d_{4 B}$ & $\mu_{1 Y}>\mu_{4 B}$ & $\mu_{1 Y}>\mu_{4 B}$ & $\mu_{1 Y}>\mu_{4 B}$ & $\sigma_{1 Y}^{2}>\sigma_{4 B}^{2}$ & $\sigma_{1 Y}^{2}>\sigma_{4 B}^{2}$ & $\sigma_{1 Y}^{2}>\sigma_{4 B}^{2}$ \\
\hline 22 & $d_{1 Y}-d_{4 G}$ & $\mu_{1 Y}>\mu_{4 G}$ & NO & NO & NO & NO & NO \\
\hline 23 & $d_{1 Y}-d_{4 Y}$ & NO & NO & NO & $N O$ & NO & NO \\
\hline 24 & $d_{1 Y}-d_{4 R}$ & NO & $\mu_{1 Y}<\mu_{4 R}$ & $N E$ & $\sigma_{1 Y}^{2}>\sigma_{4 R}^{2}$ & NO & NO \\
\hline 25 & $d_{1 R}-d_{4 B}$ & $\mu_{1 R}>\mu_{4 B}$ & $\mu_{1 R}>\mu_{4 B}$ & $\mu_{1 R}>\mu_{4 B}$ & NO & ${\sigma_{1 R}{ }^{2}>\sigma_{4 \mathrm{~B}}{ }^{2}}$ & ${\sigma_{1 R}{ }^{2}>\sigma_{4 B}{ }^{2}}^{2}$ \\
\hline 26 & $d_{1 R}-d_{4 G}$ & $N O$ & $N O$ & $N O$ & $\sigma_{1 R}^{2}<\sigma_{4 G}^{2}$ & $N O$ & ${\sigma_{1 R}}^{2}<{\sigma_{4 G}}^{2}$ \\
\hline 27 & $d_{1 R}-d_{4 Y}$ & $\mu_{1 R}<\mu_{4 Y}$ & $\mu_{1 R}<\mu_{4 Y}$ & $\mu_{1 R}<\mu_{4 Y}$ & $\sigma_{1 R}^{2}<\sigma_{4 Y}^{2}$ & NO & $\sigma_{1 R}^{2}<\sigma_{4 Y}^{2}$ \\
\hline 28 & $d_{1 R}-d_{4 R}$ & $\mu_{1 R}<\mu_{4 R}$ & $\mu_{1 R}<\mu_{4 R}$ & $\mu_{1 R}<\mu_{4 R}$ & $\sigma_{1 R}^{2}<\sigma_{4 R}^{2}$ & $\sigma_{1 R}^{2}<\sigma_{4 R}^{2}$ & NO \\
\hline
\end{tabular}

Statistically significant difference between the two datasets in the same sections was confirmed in segments BLUE, GREEN and with the exception of $\mathrm{z}$-axis for the variance in the RED segment. In the YELLOW segment, on the contrary, it is possible to say that both datasets are similar, i.e. measuring between the two identical sections $d_{1}$ and $d_{4}$. This is interesting due to different average speed, which generally indicated generation of greater shocks, which was not confirmed here despite the almost $7 \%$ higher average speed for $d_{4}$ compared to $d_{1}$.

Consistent with the findings of the individual datasets $\left(d_{1}\right.$ and $d_{4}$ ) the BLUE segment for $d_{1}$ generated on average fewer shocks than other segments for $d_{4}$. Reversely, in the BLUE segment for $d_{4}$ compared to other sections $d_{1}$, this was confirmed only for $d_{1 Y}-d_{4 B}$ and partially for $d_{1 R}-d_{4 B}$.

Generally, for individual segments (surgaces), $\mathrm{H}_{2}$ cannot be rejected due to the similarities between selected datasets (surfaces), such as YELLOW one.

\section{Results interpretation}

Evaluation of the first dataset (individual sections) shows that the BLUE segment (muddy terrain) generated, despite the common assumption, on average smaller shocks. This is primarily due to low average speed of the vehicle in the given section $3.07 \mathrm{~m} \cdot \mathrm{s}^{-1}$ in the first round or $3.77 \mathrm{~m} \cdot \mathrm{s}^{-1}$ in the last fourth round. This is based on real conditions, where, in terms of transport safety, it is not possible to move, from a certain moment, at the same speed on all types of transport routes. The 
comparison of datasets $d_{1}$ and $d_{4}$ can be interpreted analogously, where the BLUE segment (muddy terrain) for $d_{1}$ generated on average smaller shocks than all the sections for $d_{4}$. In the reverse comparison, this was confirmed for comparison with the YELLOW segment (granite cubes) for $d_{1 Y}-d_{4 B}$ and with the exception of one axis for the variance also for $d_{1 R}-d_{4 B}$. In the GREEN segment (gravel) this was only confirmed for half of the parametres (axes). However, it can be said, that despite the higher average speed, it is possible to deduce from the results that smaller shocks were generated in the BLUE segment (muddy terrain) for $d_{4}$ than in other sections $d_{1}$. Thus, to ensure safe transportation, muddy terrain generates, in the context of comparison, relatively small shocks, which on average do not exceed the normatively determined limits (for the key parameter of the arithmetic mean of the absolute values of acceleration coefficients) and also a relatively small number of extreme values occur, especially when compared to other sections. The GREEN segment (gravel) also generated on average smaller shocks than the other two sections YELLOW - granite cubes and RED - unpaved road, yet this fact was statistically confirmed only in some cases. This is mainly due to lower average speed in the GREEN segment compared to the two remaining segments (YELLOW and RED).

The recommendations for drivers and their training are analogous to those for the vehicle and transported cargo, assuming that the shocks are generally undesirable for all the mentioned categories (driver, vehicle and cargo). Their magnitude is of great importance and the recommendations are in this context as follows:

1) Driving speed and the driving style of a driver should not only comply with the basic safety principles of driving a truck, but also with the magnitude of the generated shocks, which are directly proportional to the average speed, or to aggressive driving style. Due to the higher average speed it is possible to expect greater shocks especially on transport routes of higher quality, even with the compliance with the general safety principles. The shocks may be greater than it is appropriate in relation to the driver and his health, vehicle and its life, or maintenance and service requirements and of course, in relation to cargo securing and the choice of a fastening method. Consequently, it is advisable to adjust the speed to these requirements, following the experiment results for the given vehicle type.

2) The second option is to choose a better vehicle design (tires, suspension, chassis stiffness etc.), to improve the driver's seat suspension and to choose an appropriate fastening system (primarily fasteners with higher load capacity).

The article focuses on a driver training and in connection with this, based on the experimental data, it is possible to compare not only individual drivers, e.g. whether they are aggressive drivers or rather defensive drivers [3], which is related to instant or possibly average speed and hence the magnitude of the generated shocks, but also to other requirements. Such requirements relate to the vehicle and the transported cargo. Finding the optimal driving style is therefore in such context not only safer, but also more economical. Leaving aside the less quantifiable impact on the driver's health, it is undoubtedly the impact on the life of the vehicle and the individual technical means used (e.g.straps, pallets), which entails the requirement on technical maintenance and service of a vehicle. In terms of cargo securing, it is necessary to use a fastening system with a higher load capacity or a combination of several types of fasteners, which is generally more expensive.

Specific requirements on driving characteristics of the driver during a driver training include:

- Not exceeding the normatively determined limits $\left(c_{x} \leq 0.8, c_{y}\right.$ $\leq 0.6, c_{z} \leq 2.0^{1}$ ) for the mean values (the arithmetic mean of the absolute values seems to be the most appropriate) of the measured acceleration coefficients,

- not exceeding the value \pm 1.0 for the coefficients of kurtosis and skewness with the exception of the z-axis, where the values up to +5.0 are acceptable due to the existence of extreme values (and shift of the coordinates axis),

- a percentage of values exceeding the normatively determined limits smaller than $20.0 \%$,

- a percentage of values exceeding more than double the normatively determined limits smaller than $1.0 \%$.

\section{CONCLUSION}

Modern data collection (mesurement) technologies are applicable not only onto vehicles and transport units (e.g. container units), but also onto the driving style evaluation. The application of lessons learned from the selected trasports, or driver training can be subsequently used to evaluate transports and provide a tool to guide the driving style of a given driver. Moreover, in the public sector, (e.g. the military) it is not only the economic aspect, where an inappropriate driving style causes extra costs, but also a safety aspect.

Additional costs arise as a result of the increased wear of individual parts of a vehicle, occupational diseases and damage of cargo, or other technical components used (e.g. fasteners, pallets, containers) [10].

The safety risk is related to potential damage of the abovementioned technical elements, including the vehicle, which could result in an emergency situation (e.g. traffic accident). In addition, in case of the security forces this risk may be amplified due to transport of dangerous goods (ammunition, explosives, etc.) [11].

Under military conditions such data (from accelerometers) can serve as a support to a commander's decision-making process [12]. The here presented specific requirements on a driving style also constitute a limit for ensuring safety in

\footnotetext{
1 According to CSN EN 12195-1:2011 is normatively prescribed at $c_{Z}=$ 1.0 , value 2.0 is valid for the given measuring device, where the $z$-axis is shifted by the value of gravity acceleration.
} 
relation to cargo and should substantially reduce the occurrence of the extra costs mentioned above.

Further research will focus on application of more sophisticated statistical (e.g. [13]) and other methods, such as spectral analysis (e.g. [14, 15]) or extreme value theory [16]. The tools of economical analysis [17] or urban planning [18, 19] can be applied as well.

The prerequisite for further research is also the evaluation of shocks in other modes of transport (rail, air, water) to optimize the operation of particular means of transport for cargo transport.

The results of the analyses can also be applied to other areas, such as agricultural and forestry technology [20], or the Integrated Rescue System.

\section{ACKNOWLEDGMENT}

The paper was written with the support of the project of long-term strategy of organization development: ROZVOLOG: Development of Capabilities and Sustainability of Logistics Support (DZRO ROZVOLOG 2016-2020) funded by the Ministry of Defence of the Czech Republic.

\section{REFERENCES}

[1] P. Foltin et al. "Discrete Event Simulation in Future Military Logistics Applications and Aspects (Published Conference Proceedings style)", in Proc. of Modelling and Simulation for Autonomous Systems Conf, Roma, 2017, pp. 410-422.

[2] K. Bucsuhazy et al. "Analysis of Driver Reaction Time Using the Acquisition of Biosignals (Published Conference Proceedings style)", in Proc. of $3^{\text {rd }}$ Int. Conf. on Traffic and Transport Engineering - ICTTE Belgrade, Belgrade, 2016, pp. 68-74.

[3] M. Vlkovsky and M. Smerek. "Statistical Analysis of Driving Style and its Effect on Cargo Securing (Published Conference Proceedings style)", in Proc. of $4^{\text {th }}$ Int. Conf. on Traffic and Transport Engineering - ICTTE Belgrade, Belgrade, 2018, pp. 744-750.

[4] M. Vlkovsky et al. "Cargo Securing during Transportation - Using Extreme Values (Published Conference Proceedings style)", in Proc. of $23^{\text {rd }}$ Int. Conf. The Knowledge-Based Organization: Applied Technical Sciences and Advanced Military Technologies, Sibiu, 2017, pp. 142147.

[5] CSN EN 12195-1. Restraining on Road Vehicles - Safety - Part 1: Calculation of Securing Forces. Czech Office for Standards, Metrology and Testing: Prague, 2010.

[6] European Commision - Directorate-General for Energy and Transport (2016) European Best Practice Guidelines on Cargo Securing for Road Transport. [online]. Available from: www.uirr.com/fr/component/ downloads/downloads/302.html

[7] T. Lerher. Cargo Securing in Road Transport Using Restraining Method with Top-over Lashing. Nova: New York, 2015. ISBN 978-161122-002-5.

[8] G. Grossmann and M. Kassmann. Safe Packaging and Load Securing in Transport. $3^{\text {rd }}$ Ed. Expert Verlag: Renningen, 2018. ISBN: 978-38169-3334-2.

[9] V. Kolmas et al. Catalog of Automotive and Tracked Vehicles Used in the ACR. Ministry of Defence of the Czech Republic - AVIS: Prague, 2007. ISBN 978-80-7278-382-3.

[10] M. Vlkovsky et al. "Cargo Securing and its Economic Consequences (Published Conference Proceedings style)", in Proc. of $22^{\text {nd }}$ Int. Scientific Conf. Part I - Transport Means, Kaunas, 2018, pp. 129-135.

[11] M. Vlkovsky and L. Rak, "Cargo Securing in Selected Vehicles and Transport of Explosives (Periodical style)", Perner's Contacts, vol. 12, no. 3, pp. 101-110, 2017.

[12] O. Rolenec et al., "Supporting the decision-making process in the planning and controlling of engineer task teams to support mobility in a combat operation (Periodical style)", International Journal of
Education and Information Technologies, vol. 2019, no. 13, pp. 33-40, 2019.

[13] M. Vlkovsky et al. "Impact of Shocks on Cargo Securing During the Road Transport (Published Conference Proceedings style)", in IOP Conference Series - Materials Science and Engineering of $4^{\text {th }}$ World Multidisciplinary Civil Engineering, Architecture, Urban Planning Symposium, Prague, 2019.

[14] M. Vlkovsky et al. "Wavelet Based Analysis of Truck Vibrations during Off-road Transportation (Published Conference Proceedings style)", in Proceedings MATEC Web of Conferences of $14^{\text {th }}$ International Conference on Vibration Engineering and Technology of Machinery, Lisabon, 2018.

[15] D. Grzesica. "Measurement and Analysis of Truck Vibrations during Off-road Transportation (Published Conference Proceedings style)", in Proceedings MATEC Web of Conferences of $14^{\text {th }}$ International Conference on Vibration Engineering and Technology of Machinery, Lisabon, 2018.

[16] J. Cavadas et al., "Road Safety of Passing Maneuvers: A Bivariate Extreme Value Theory Approach under Non-Stationary Conditions", Accident Analysis and Prevention, vol. 134, 2020.

[17] A. Carteni, "A Cost-Benefit Analysis Based on the Carbon Footprint Derived from plug-in Hybrid Electric Buses for Urban Public Transport Services", in WSEAS Transactions on Environment and Development, vol. 14, pp. 125-135, 2018.

[18] H. Largo et al., "Public Transportation Demand Model for Low Density Territories", in WSEAS Transactions on Environment and Development, vol. 15, pp. 395-407, 2019.

[19] M. Botte et al., "A Geometrical Approach to Reduce Calculation Times in the Definition of Rail Convoy Speed Profiles", in WSEAS Transactions on Environment and Development, vol. 16, pp. 98-104, 2020

[20] M. Vlkovsky and P. Veselik, "Cargo Securing - Comparison of Different Quality Roads (Periodical style)", Acta Universitatis Agriculturae et Silviculturae Mendelianae Brunensis, vol. 67, no. 4, pp. 1015-1023, 2019.

MAJ Assoc. Prof. Martin Vlkovský, PhD, born in 1981, graduated from the University of Defense in Brno, in the "Defence Economy" (modul "Logistics"). Doctoral degree (PhD) gained at the University of Defence in Brnno in the "Defence Economy" focusing on logistics and transport as well. He defended his associate professor thesis in the "Military Management" at the same university in 2019. He deals with logistics and trasportation issues, particulary with cargo securing during transport and material handling operations. 